\title{
Huaqing Wang, China CDC's Chief Expert in National Immunization Program
}

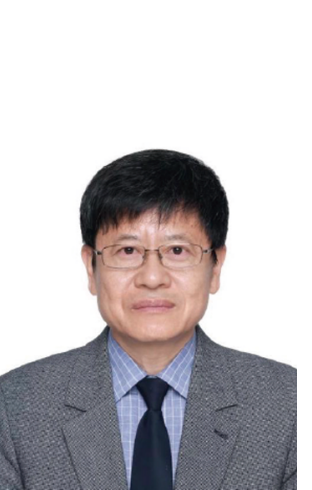

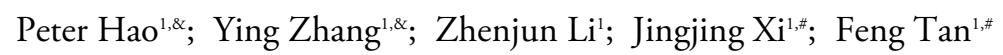

Huaqing Wang has had a long and distinguished career in developing and implementing immunization programs to protect China's people. After graduating from Harbin Medical University in 1986 with a major in preventive medicine, Huaqing Wang was assigned to Heilongjiang Provincial CDC and has dedicated his career to infectious disease control and immunization.

In 1986, China's National Immunization Program (NIP) was experiencing rapid development and progress as multiple sectors of public health workers were hard at work achieving the first nationwide coverage goal of at least $85 \%$ of children in every provinciallevel administrative division (PLAD) receiving all NIP vaccines. The Chinese government prioritized childhood immunization with the State Council of China approving and establishing the NIP and specifying April 25 every year as "National Child Vaccination Day." With the government's support and sponsorship from the United Nations International Children's Fund (UNICEF), China's vaccine cold chain — a system of storing and transporting vaccines at recommended temperatures to preserve the vaccines' efficacy — was established and perfected. Simultaneously, immunization services were gradually being aligned with international technical standards with the support of the World Health Organization (WHO).

By the end of 1986, Huaqing Wang became greatly impressed by an epidemiological investigation of poliomyelitis that caused him to become engaged with the NIP for over 30 years. In the winter of 1986-1987, Huaqing Wang and his senior colleagues discovered that many children with polio had not been reported or were misreported during local CDC investigations. The issue was significant and urgent as poliomyelitis could cause lifelong disability or, in severe cases, could cause patients and survivors to lose any ability to care for themselves as no medications can treat polio, leaving immunization as the only strategy capable of stopping polio from harming children. In Qitaihe City of Heilongjiang Province, Huaqing Wang saw a 2-year-old boy suffering from severe poliomyelitis and having extremely limited muscle strength — grade 1 to 2 out of 4 . This child being immobile and bedridden due to missed or unavailable poliovirus vaccinations left an important image in his heart and mind and inspired his continuing work with the NIP.

The NIP continued to focus on vaccine accessibility and elimination of polio, and China successively achieved planned targets of $85 \%$ childhood coverage with NIP vaccines at the provincial-level in 1988, the county-level in 1990, and the township-level in 1995. In 2000, China was certified as polio-free by the WHO Western Pacific Region, and Huaqing Wang received individual honors from China NIP in 1999 and was recognized as a National Advanced Polio Eradication Leader in 2001.

In addition to this domestic work, Huaqing Wang also studied advanced molecular biology for one year with the virology group in the Department of Medicine of Niigata University in Japan to enrich his knowledge and understanding of molecular biology in 1998. Following this, he obtained a master's degree in epidemiology from 1999 to 2001 and began doctoral studies on disease etiology at the University of Tokushima in Japan in 2002. By 2005, Huaqing Wang was transferred to the Department of the National Immunization Program of the recently established China CDC and had several major accomplishments.

As the technical director, Huaqing Wang introduced the ideas and methods of evidence-based medicine into China's Expanded Program on Immunization (EPI 2007) and included evidence-based recommendations for a live, attenuated Japanese-encephalitis vaccine and hepatitis A vaccine, both of which were independently developed by China. He and a group of senior colleagues also led the development of China's Adverse Events Following Immunization (AEFI) Monitoring System from 2011 to 2014, which eventually passed WHO assessments to allow 
vaccines produced in China to be internationally recognized. In addition, over 100 national-level programs and guidelines such as the widely used "Work Specifications for Vaccinations" were developed under his leadership and guidance.

Beyond this work, Huaqing Wang also personally leads responses to public health events. Media coverage of vaccines can sometimes cause worry and doubt in recipients, parents, or general citizens and require a disciplined, scientific response to curb harm to societal perception of vaccines. For instance, the Shanxi Province "high temperature" incidents in 2008 and 2010, the measles vaccine incident in 2010, the hepatitis B vaccination child death incident in 2013, and the Shandong Province "illegal" vaccine incident in 2016 showcased the continued need for professionals to carry out cohort investigations and causal inference analysis on deaths, diseases, or symptoms that are related with vaccines. The technical support for the conclusion of the vaccine incidents is critical for maintaining public trust and averting panic.

Huaqing Wang also directs response to emerging and existing outbreaks of infectious diseases. In 2009, Huaqing Wang also led the development and implementation of the "Guidance for the Clinical Trial of H1N1 Influenza Vaccine (2009, China)" helping China become the first country to launch an H1N1 influenza vaccine. In addition, during a polio epidemic in Xinjiang Uyghur Autonomous Region, he and a team of senior colleagues led joint analyses of polio surveillance that examined the main population, age structure, regional distributions, and time distribution of polio cases to yield an epidemiologically-based emergency immunization strategy. The adopted strategy included specified areas, age ranges, vaccination timing, and dosing and was precise enough to require only two months to control the epidemic. Later, the WHO recognized this response as a model for global control of polio.

As the Chief Expert for Immunization Program of China CDC, Huaqing Wang plays a crucial role in guiding China's immunization programs, in responding to outbreaks and emergencies, in technical analysis, and in provision of immunization services in disasters.

doi: $10.46234 / \mathrm{ccdcw} 2020.154$

\# Corresponding authors: Jingjing Xi, xijj@chinacdc.cn; Feng Tan, tanfeng@chinacdc.cn.

${ }^{1}$ Chinese Center for Disease Control and Prevention, Beijing, China.

\& Joint first authors.

Submitted: July 15, 2020; Accepted: July 20, 2020 